\title{
Vacuum Fluctuations of Energy Density can lead to the observed Cosmological Constant
}

\author{
T. Padmanabhan \\ Inter-University Centre for Astronomy and Astrophysics, Post Bag 4, \\ Ganeshkhind, Pune-411 00\%. email: nabhan@iucaa.ernet.in
}

\begin{abstract}
The energy density associated with Planck length is $\rho_{u v} \propto L_{P}^{-4}$ while the energy density associated with the Hubble length is $\rho_{i r} \propto L_{H}^{-4}$ where $L_{H}=1 / H$. The observed value of the dark energy density is quite different from either of these and is close to the geometric mean of the two: $\rho_{v a c} \simeq \sqrt{\rho_{u v} \rho_{i r}}$. It is argued that classical gravity is actually a probe of the vacuum fluctuations of energy density, rather than the energy density itself. While the globally defined ground state, being an eigenstate of Hamiltonian, will not have any fluctuations, the ground state energy in the finite region of space bounded by the cosmic horizon will exhibit fluctuations $\Delta \rho_{\mathrm{vac}}\left(L_{P}, L_{H}\right)$. When used as a source of gravity, this $\Delta \rho$ should lead to a spacetime with a horizon size $L_{H}$. This bootstrapping condition leads naturally to an effective dark energy density $\Delta \rho \propto\left(L_{u v} L_{H}\right)^{-2} \propto H^{2} / G$ which is precisely the observed value. The model requires, either (i) a stochastic fluctuations of vacuum energy which is correlated over about a Hubble time or (ii) a semi-anthropic interpretation. The implications are discussed.

PACS numbers:
\end{abstract}

The conventional discussion of the relation between cosmological constant and vacuum energy density is based on evaluating the zero point energy of quantum fields with an ultraviolet cutoff and using the result as a source of gravity. Any reasonable cutoff will lead to a vacuum energy density $\rho_{\text {vac }}$ which is unacceptably high [1].

This argument, however, is too simplistic since the zero point energy - obtained by summing over the $(1 / 2) \hbar \omega_{k}$ - has no observable consequence in any other phenomena and can be subtracted out by redefining the Hamiltonian. The observed non trivial features of the vacuum state of QED, for example, arise from the fluctuations (or modifications) of this vacuum energy rather than the vacuum energy itself. This was, in fact, known fairly early in the history of cosmological constant problem and, in fact, is stressed by Zeldovich 2] who explicitly calculated one possible contribution to fluctuations after subtracting away the mean value. This suggests that we should consider the fluctuations in the vacuum energy density in addressing the cosmological constant problem.

Similar viewpoint arises, more formally, when we study the question of detecting the energy density using gravitational field as a probe. Recall that an Unruh-DeWitt detector with a local coupling $L_{I}=M(\tau) \phi[x(\tau)]$ to the field $\phi$ actually responds to $\langle 0|\phi(x) \phi(y)| 0\rangle$ rather than to the field itself 3 . Similarly, one can use the gravitational field as a natural "detector" of energy momentum tensor $T_{a b}$ with the standard coupling $L=\kappa h_{a b} T^{a b}$. Such a model was analysed in detail in ref. [4] and it was shown that the gravitational field responds to the two point function $\left\langle 0\left|T_{a b}(x) T_{c d}(y)\right| 0\right\rangle$. In fact, it is essentially this fluctuations in the energy density which is computed in the inflationary models [5] as the seed source for gravitational field, as stressed in ref. [6]. All these suggest treating the energy fluctuations as the physical quantity "detected" by gravity, when one needs to incor- porate quantum effects.

This fact alone, however, would not have helped. The vacuum expectation values in question are all ultraviolet divergent and will - normally - scale as a suitable power of the UV-cutoff, $L_{u v}$. If the energy density behaves as $\rho \propto L_{u v}^{-4}$, the fluctuation $\Delta \rho$ will also scale as $L_{u v}^{-4}$ since that is the dominant scale in the problem; so the cosmological constant will still come out too large. Another difficulty is that, at least formally, the ground state is an energy eigenstate and it will have no dispersion in the energy. However, as we shall describe, there is a nice manner in which De-Sitter spacetime circumvents these difficulties and leads to the correct value for the cosmological constant.

The key new ingredient arises from the fact that the properties of the vacuum state (or, for that matter, any quantum state in field theory) depends on the scale at which it is probed. It is not appropriate to ask questions without specifying this scale. (This, in some sense, has been the key lesson from renormalisation group.) If the spacetime has a cosmological horizon which blocks information, the natural scale is provided by the size of the horizon, $L_{H}$, and we should use observables defined within the accessible region. The operator $H\left(<L_{H}\right)$, corresponding to the total energy inside a region bounded by a cosmological horizon, will exhibit fluctuations $\Delta E$ since vacuum state is not an eigenstate of this operator. The corresponding fluctuations in the energy density, $\Delta \rho \propto(\Delta E) / L_{H}^{3}=f\left(L_{u v}, L_{H}\right)$ will now depend on both the ultraviolet cutoff $L_{u v}$ as well as $L_{H}$. We now see an interesting possibility of boot strapping: When used as the source of gravity, this $\Delta \rho$ should lead to a spacetime with the horizon size $L_{H}$, which, in turn, requires us to compute $\Delta \rho$ using $L_{H}$ as the infrared cutoff scale. Note that it is the existence of a cosmological horizon at $L_{H}$ which provides a clear justification for using this length scale in computing the energy fluctuations. This 
leads to a value for $\Delta \rho$ different from $L_{u v}^{-4}$ since we now have two length scales in the problem. This bootstrapping will lead to a relation between $\Delta \rho, L_{u v}$ and $L_{H}$. We will show that this relation is $\Delta \rho \propto\left(L_{u v} L_{H}\right)^{-2} \propto H^{2} / G$ if we take the ultraviolet cut off at Planck length. That is precisely what we need.

Remarkably enough, this result is very easy to obtain. To obtain $\Delta \rho_{\text {vac }} \propto \Delta E / L_{H}^{3}$ which scales as $\left(L_{P} L_{H}\right)^{-2}$ we need to have $(\Delta E)^{2} \propto L_{P}^{-4} L_{H}^{2}$; that is, the square of the energy fluctuations should scale as the surface area of the bounding surface which is provided by the cosmic horizon. A simple argument to show that such a scaling is likely to occur is the following. Let the total Hamiltonian $H$ of the system be written as $H=H_{1}+H_{2}$ where $H_{1}$ is the Hamiltonian obtained by integrating the Hamiltonian density within a sphere of radius $R\left(=L_{H}\right)$ and $H_{2}$ denoting the Hamiltonian of the outside region. (We are dividing the real space rather than separating scales in the momentum space, as is done in standard RG analysis.) Let $E_{1}$ and $E_{2}$ be the expectation values of $H_{1}$ and $H_{2}$ in the ground state $|0\rangle$ which is taken to be an eigen state of $H$ with $H|0\rangle=E|0\rangle$. Consider now the energy dispersions in the regions 1 and 2. Writing $\left(H_{2}-E_{2}\right)^{2}$ as $\left[(H-E)-\left(H_{1}-E_{1}\right)\right]^{2}$ and noticing that the expectation values of $(H-E)$ and $(H-E)^{2}$ vanish in any energy eigenstate, we get the result

$$
\left(\Delta E_{2}\right)^{2} \equiv\left\langle 0\left|\left(H_{2}-E_{2}\right)^{2}\right| 0\right\rangle=\left\langle 0\left|\left(H_{1}-E_{1}\right)^{2}\right| 0\right\rangle=\left(\Delta E_{1}\right)^{2}
$$

That is, the dispersions in the energy in the regions 1 and 2 are equal. Since the regions 1 and 2 only share the bounding surface, this relation suggests that either dispersion could be proportional to the area of the surface and will scale as $R^{2}$. The dependence on $L_{P}$ is then fixed by dimensional considerations and we get $(\Delta E)^{2} \propto$ $L_{P}^{-4} R^{2}$.

While the above argument is suggestive, it leaves significant scope for improvement - not in the least because one is dealing with formally divergent quantities. Fortunately, it is possible to do this calculation rigorously. In flat spacetime, a scalar field has the familiar mode expansion

$$
\phi(x)=\int \frac{d^{3} k}{(2 \pi)^{3}} q_{\mathbf{k}}(t) e^{i \mathbf{k} \cdot \mathbf{x}}
$$

in terms of the harmonic oscillator modes $q_{\mathbf{k}}(t)$. Expressing the Hamiltonian in the region 1

$$
H_{1}=\int_{|x|<L_{H}} d^{3} \mathbf{x} \frac{1}{2}\left(\dot{\phi}^{2}+|(\nabla \phi)|^{2}\right)
$$

in terms of $\dot{q}_{\mathbf{k}}$ and $q_{\mathbf{k}}$ one can evaluate the dispersion

$$
(\Delta E)^{2}=\left\langle 0\left|\left(H_{1}-\left\langle H_{1}\right\rangle\right)^{2}\right| 0\right\rangle
$$

in a straightforward manner. To obtain a finite result, one needs to use an ultra violet regulator which we take to be the Planck length. For $L_{H} \gg L_{P}$, the final result has the scaling

$$
(\Delta E)^{2}=c_{1} \frac{L_{H}^{2}}{L_{P}^{4}}
$$

where the constant $c_{1}$ depends on the manner in which ultra violet cutoff is imposed. Similar calculations have been done (with a completely different motivation, in the context of entanglement entropy) by several people and it is known that the area scaling found in Eq. (5), proportional to $L_{H}^{2}$, is a generic feature [7]. For a simple exponential UV-cutoff, $c_{1}=\left(1 / 30 \pi^{2}\right)$ but we do not believe this can be computed reliably without knowing the full theory. The result should also scale with the total number of degrees of freedom at sub-Planckian energies which is not reliably known. [It is amusing to note that, the standard Zeldovich-Harrison scale invariant spectrum has the mass fluctuation $(\delta M / M)^{2} \propto k^{3} P(k) \propto k^{4} \propto R^{-4}$ leading to the same "area" scaling $(\delta M)^{2} \propto R^{2}$.]

We thus find that the fluctuations in the energy density of the vacuum in a sphere of radius $L_{H}$ is given by

$$
\Delta \rho_{\mathrm{vac}}=\frac{\Delta E}{L_{H}^{3}} \propto L_{P}^{-2} L_{H}^{-2} \propto \frac{H^{2}}{G}
$$

The numerical coefficient will depend on $c_{1}$ as well as the precise nature of infrared cutoff radius (like whether it is $L_{H}$ or $L_{H} / 2 \pi$ etc.). It would be pretentious to cook up the factors to obtain the observed value for dark energy density.

The current result in which the fluctuations in the energy density act as a source of gravity is similar in spirit to the idea presented earlier in ref. [8]. If a quantum system is in a stationary state, its phase will evolve as $\exp (-i E t)$. But for a partial system which can undergo fluctuations in the energy, one cannot attribute such a phase and we will - instead - obtain an uncertainty relation of the kind $\Delta E \propto(1 / \tau)$ where $\tau$ is the time scale of the fluctuations. Similarly, the semiclassical gravity will have a wave function with a phase $\exp \left(-i \rho_{\text {vac }} \mathcal{V}\right)$ in case of a constant vacuum energy density, where $\mathcal{V}$ is the four volume. In case of a finite region of space, the fluctuations lead to the result $\Delta \rho_{\mathrm{vac}} \propto\left(L_{H} L_{P}\right)^{-2}$. To be rigorous, we need to do this computation in the DeSitter geometry rather than in flat spacetime. There is no conceptual difficulty in carrying out this program which merely requires replacing the mode functions in Eq. (2) by the ones appropriate for De-Sitter spacetime. It is easy to see from dimensional considerations that the results in Eq. (5) and Eq. (6) will continue to hold. The numerical factor changes and needs to be determined by numerical integration.

We have concentrated on the energy density rather than on the full energy momentum tensor for the sake of simplicity. More formally, one can construct the fluctuations in $T_{a b}$ along the same lines thereby obtaining the equation of state for the dark energy. If the regularization of the divergent expressions is handled in a Lorentz invariant manner (like, for example, using dimensional 
regularization in curved spacetime), then the Lorentz invariance dictates that the final result should have the form $\Delta T_{a b}=\Delta \rho_{\mathrm{vac}} g_{a b}$.

The current observations ( $[9]$; for earlier indications of nonzero cosmological constant, see [10] ) do suggest that

$$
\rho_{v a c} \simeq \sqrt{\rho_{U V} \rho_{I R}}
$$

if we take the UV scale as Planck scale and the IR scale as Hubble scale. This remarkable relation deserves an explanation which is provided in a simple manner by our analysis. In fact, if we start with this result and do a bit of "reverse-engineering", we immediately obtain the area scaling of energy fluctuations. The latter has been pursued and obtained by several authors, in connection with the holographic ideas and entropy-area connection.

The main criticism one could raise is regarding our assumption that we can ignore the mean energy density, regulated by the UV cut off to a large value and concentrate on its fluctuation. In addition to the arguments given in the first paragraph of this paper, there is another aspect which need to be stressed: It is a fact of life that a fluctuation of magnitude $\Delta \rho_{v a c} \simeq H^{2} / G$ will exist in the energy density inside a sphere of radius $H^{-1}$ if Planck length is the UV cut off. One cannot get away from it. On the other hand, observations suggest that there is a $\rho_{v a c}$ of similar magnitude in the universe. It seems natural to identify the two, after subtracting out the mean value by hand. Our goal was more towards explaining why there is a surviving cosmological constant which satisfies Eq. (7) which - in our opinion - is the problem. Another technical objection could be regarding our computing the $\Delta E$ over a sphere of radius $H^{-1}$ and dividing it by the volume to get $\Delta \rho_{\text {vac }}$. This corresponds to assuming the coherence scale of fluctuation to be Hubble radius, which is indeed the correct thing to do. Recall that it is the horizon at $H^{-1}$ which motivates choosing this scale rather than some other smaller scale.

How does one interpret this result ? There are two possible ways. The first is to claim that the currently observed value of cosmological constant has no special significance and the fluctuations will lead to a $\rho_{v a c} \approx$ $H^{2}(t) / G$ at any epoch. This suggests a stochastic interpretation of cosmological constant. To obtain the evolution of the universe, we now need to solve the Friedmann equations with $\rho_{v a c}$ being a stochastic variable with a probability distribution $\mathcal{P}\left(\rho_{\text {vac }}\right)$ which can also be computed in the ground state. Since the energy fluctuations can be positive or negative, in general, the cosmological constant can have either sign. There are (at least!) two difficulties in this interpretation:

(i) For this idea to be viable, we need the fluctuations to be correlated over a time scale of the order of $H(t)^{-1}$. It is trivial to compute the two point function $C(t)=\left\langle 0\left|H_{1}\left(t_{1}\right) H_{1}\left(t_{1}+t\right)\right| 0\right\rangle$ for the quantum fluctuations and one finds that $C(t)=c_{2}\left(L_{H}^{2} t^{-4}\right)$; this quantity is finite and we do not need to introduce the UV cutoff. (If the cutoff is introduced the result is
$\left.C(t) \propto L_{H}^{2}\left(L_{P}^{2}+t^{2}\right)^{-2}\right)$. Being a power law correlation, this suggests fluctuations at all time scales with the scale governed by $L_{H}$. But when $t \approx L_{H}$, the ratio $\left[C\left(L_{H}\right) / C(0)\right]^{1 / 2}=\left(L_{P} / L_{H}\right)^{2} \ll 1$ if $C(0)$ is computed with a cut off.. This probably means that coherence over $t \approx L_{H}$ is rare. But the argument is not conclusive since what is required is the coherence time, given that a fluctuation of order $L_{H}^{2} / L_{P}^{4}$ has occurred. If we assume that coherence over $t \approx L_{H}$ is possible, then the current positive value of cosmological constant is a result of a fluctuation lasting typically for a time scale of $H_{0}^{-1}$ and we would predict to see a reversal of sign of $\rho_{v a c}$ at earlier epochs. (Except that, it is difficult to predict whether this should have occurred at $z=2$ or at $z=2 \pi^{2}$, say, with any confidence until all the numerical factors are accounted for. On the other hand, if the SN data at a redshift of 2, say, does indicate a negative cosmological constant, ones faith in this stochastic fluctuations will increase!). With this interpretation, the model solves both the conventional "problems" of the cosmological constant. Its value is correctly explained as due to energy fluctuations and this relation holds at any epoch over a local time scale of $H(t)^{-1}$ thereby answering "why now?" by "always!".

(ii) The second difficulty is is not special to our model and arises whenever one tries to model quantum fluctuations as a classical stochastic process and treat it as a source of semiclassical gravity. In the standard cosmological model, the consistency of $\left(\begin{array}{l}0 \\ 0\end{array}\right)$ component of Einstein's equation with the $\left(\begin{array}{l}1 \\ 1\end{array}\right)$ component will demand that $d\left(\rho a^{3}\right)=-p d\left(a^{3}\right)$ which in turn will require $\rho$ to be independent of time if $p=-\rho$. This relation will require a reinterpretation when cosmological constant is treated as a stochastic variable. If the coherence time of the fluctuation is of the order of Hubble time, there will be constancy of effective $\rho_{v a c}$ (and standard evolution of the universe) within one Hubble time. But when stochastic fluctuation of cosmological constant occurs one needs to interpret the energy conservation equation in some limiting form.

Other issues that one could raise regarding the computation in this approach are relatively straight forward to address: one can work out the probability distribution for fluctuations in $T_{a b}$ in a De-Sitter background and integrate the stochastic Friedman equation. One also need to study the effect of the cosmological constant fluctuations on the growth of structures. All of these are best addressed numerically since one need to deal with a stochastic process with finite correlation.

There is a completely different way of interpreting this result based on some imaginative ideas suggested by Bjorken 11] recently. The key idea here is to parametrise the universes by the value of $L_{H}$ which they have. It is a fixed, pure number for each universe in an ensemble of universes but all the other parameters of the physics are correlated with $L_{H}$. This is motivated by a series of arguments in ref. 11] and, in this approach, $\rho_{\text {vac }} \propto L_{H}^{-2}$ almost by definition; the hard work was in determining how other parameters scale with $L_{H}$. In the approach 
suggested here, a dynamical interpretation of the scaling $\rho_{\text {vac }} \propto L_{H}^{-2}$ is given as due to vacuum fluctuations of other fields. We now reinterpret each member of of the ensemble of universes as having zero energy density for vacuum (as any decent vacuum should have) but the effective $\rho_{v a c}$ arises from the quantum fluctuations with the correct scaling. One can then invoke standard anthropiclike arguments (but with very significant differences as stressed in ref. [1] ) to choose a range for the size of our universe. This appears to be much more attractive way of interpreting the result than introducing stochastic, time dependent fluctuations.
Finally, to be fair, this attempt should be judged in the backdrop of other suggested solutions almost all of which require introducing extra degrees of freedom in the form of scalar fields, modifying gravity or introducing higher dimensions etc. and fine tuning the potentials (for a non-comprehensive sample of references, see [12].) At a fundamental level such approaches are unlikely to provide the final solution.

I thank K.Subramanian for several rounds of extensive discussions and R.Nityananda and A.D.Patel for useful comments on previous drafts.
[1] For reviews, see Weinberg, S, Rev. Mod. Phys., 61, 1(1989); P. J. E. Peebles and B. Ratra, Rev. Mod. Phys. 75, 559 (2003); T. Padmanabhan, Phys. Rept. 380, 235 (2003);

[2] Zel'dovich, Y.B., JETP letters 6, 316 (1967); Soviet Physics Uspekhi 11, 381 (1968).

[3] Unruh, W. G.,1976, Phys. Rev.,D14, 870; DeWitt, B. S., in General Relativity: An Einstein Centenary Survey, pp680-745 Cambridge University Press,1979 ed., S.W. Hawking and W. Israel; T. Padmanabhan,Class. Quan. Grav. , (1985), 2 , 117; L. Sriramkumar and T. Padmanabhan, Int. Jour. Mod. Phys. D 11,1 (2002) [gr-qc9903054]

[4] T.Padmanabhan and T.P. Singh, Class. Quan. Grav. , (1987), $4,1397$.

[5] S. W. Hawking, Phys. Lett. B 115, 295 (1982); A. H. Guth and S.-Y. Pi, Phys. Rev. Lett. 49, 1110 (1982); J. M. Bardeen, P. J. Steinhardt and M. S. Turner, Phys. Rev. D 28, 679 (1983), T.Padmanabhan , Phys. Rev. Letts. , 1988, 60 , 2229.

[6] T.Padmanabhan, T.R. Seshadri and T.P. Singh, Phys. Rev. D .(1989) $39,2100$.

[7] L. Bombelli, R. K. Koul, J.-H. Lee, and R. D. Sorkin, Phys. Rev. D34, 373 (1986); M. Srednicki, Phys. Rev. Lett. 71, 666 (1993), hep-th/9303048 R. Brustein, D. Eichler, S. Foffa, and D. H. Oaknin, Phys. Rev. D65, 105013 (2002), hep-th/0009063 A. Yarom, R. Brustein, hep-th/0401081 and references cited therein. Our results can also be obtained easily from those in ref. [4].

[8] T. Padmanabhan , Class.Quan.Grav. , 19, L167 (2002), gr-qc/0204020; for an earlier attempt, see D. Sorkin, 1997 Forks in the road, on the way to quantum gravity, gr-qc/9706002; for an interesting alternative view see, Volovik, G. E., gr-qc/0405012

[9] J. L. Tonry et al., ApJ 594, 1 (2003); B. J. Barris, astro-ph/0310843 A. G.Reiss et al., astro-ph/0402512 (2004); A. G. Riess et al., astro-ph/0402512; A. Melchiorri et al. [Boomerang Collaboration], Astrophys. J. 536, L63 (2000). D. N. Spergel et al., ApJS 148, 175 (2003); for a recent data analysis, seeT. Padmanabhan and T. Roy Choudhury, Mon. Not. Roy. Astron. Soc. 344, 823 (2003); T. Roy Choudhury and T. Padmanabhan, astro-ph/0311622 ; H.K.Jassal, J.S.Bagla, T. Padmanabhan astro-ph/0404378.

[10] G. Efstathiou, W. J. Sutherland and S. J. Maddox, Nature 348, 705 (1990); J. P. Ostriker and P. J. Steinhardt, Nature 377, 600 (1995); J. S. Bagla, T. Padmanabhan and J. V. Narlikar, Comments on Astrophysics 18, 275 (1996).

[11] J.D. Bjorken, Phys.Rev. D67 (2003) 043508; astro-ph/0404233

[12] J. R. Ellis, Phil. Trans. Roy. Soc. Lond. A 361, 2607 (2003). P. J. Steinhardt, Phil. Trans. Roy. Soc. Lond. A 361, 2497 (2003); P. F. Gonzalez-Diaz, Phys. Rev. D 62, 023513 (2000); C. Armendariz-Picon, V. Mukhanov and P. J. Steinhardt, Phys. Rev. D 63, 103510 (2001); T. Padmanabhan, Phys. Rev. D 66, 021301 (2002); J. S. Bagla, H. K. Jassal and T. Padmanabhan, Phys. Rev. D 67, 063504 (2003); T. Padmanabhan and T. R. Choudhury, Phys. Rev. D 66, 081301 (2002);G. W. Gibbons, Class. Quant. Grav. 20, S321 (2003); R. R. Caldwell, Phys. Lett. B 545, 23 (2002);J. g. Hao and X. z. Li, Phys. Rev. D 67, 107303 (2003). 